\title{
New insights into protein-protein interaction data lead to increased estimates of the $S$. cerevisiae interactome size
}

\author{
Laure Sambourg, Nicolas Thierry-Mieg*
}

\begin{abstract}
Background: As protein interactions mediate most cellular mechanisms, protein-protein interaction networks are essential in the study of cellular processes. Consequently, several large-scale interactome mapping projects have been undertaken, and protein-protein interactions are being distilled into databases through literature curation; yet protein-protein interaction data are still far from comprehensive, even in the model organism Saccharomyces cerevisiae. Estimating the interactome size is important for evaluating the completeness of current datasets, in order to measure the remaining efforts that are required.

Results: We examined the yeast interactome from a new perspective, by taking into account how thoroughly proteins have been studied. We discovered that the set of literature-curated protein-protein interactions is qualitatively different when restricted to proteins that have received extensive attention from the scientific community. In particular, these interactions are less often supported by yeast two-hybrid, and more often by more complex experiments such as biochemical activity assays. Our analysis showed that high-throughput and literaturecurated interactome datasets are more correlated than commonly assumed, but that this bias can be corrected for by focusing on well-studied proteins. We thus propose a simple and reliable method to estimate the size of an interactome, combining literature-curated data involving well-studied proteins with high-throughput data. It yields an estimate of at least 37, 600 direct physical protein-protein interactions in S. cerevisiae.

Conclusions: Our method leads to higher and more accurate estimates of the interactome size, as it accounts for interactions that are genuine yet difficult to detect with commonly-used experimental assays. This shows that we are even further from completing the yeast interactome map than previously expected.
\end{abstract}

\section{Background}

As the chief actors within the cell, proteins participate in every cellular process, from metabolism to mechanical structure, immune system or signaling pathways. To successfully fulfill their role, they stably or transiently interact with each other, forming a complex protein interaction network, or interactome. Thus, the comprehensive mapping and deciphering of theses interactomes is a prerequisite for the full understanding of any cellular system. Furthermore, interactomes can be used to infer the function and regulation of novel proteins (e.g. Tarassov et al. predict that the previously uncharacterized proteins YML018C, YMR221C and YDR119W are

\footnotetext{
* Correspondence: Nicolas.Thierry-Mieg@imag.fr

Laboratoire TIMC-IMAG, BCM, CNRS UMR5525, Faculté de médecine, 38706
} La Tronche cedex, France involved in autophagy [1]). However, when trying to extract information from protein interaction networks, one must be aware that they are far from comprehensive. Estimating the size of an interactome provides insight into the biological relevance of the conclusions drawn. For example, partial sampling from networks presenting a variety of degree distributions can result in apparent scale-free subnetworks, irrespective of the initial network's topology [2]: topology analyses based on incomplete data may not be valid. Moreover, the number of protein-protein interactions is an important parameter for evaluating the completeness of databases and current high-throughput experiments, in order to measure the remaining efforts and build a framework for future experiments $[3,4]$. We focus here on S. cerevisiae, one of the most studied eukaryotic model

\section{() Biomed Central}


organisms and a widely-used test platform for new experimental techniques, in particular for proteinprotein interaction (PPI) detection methods.

\section{Available data}

The available datasets of protein-protein interactions fall into two categories: literature-curated (LC) and highthroughput (HT). LC data reports manually curated interactions described in the literature, usually obtained by low-throughput experiments [5]. While high-throughput datasets are typically produced by testing all pairs of proteins within a subspace determined solely by the availability of reagents, low-throughput experiments are often hypothesis-driven, for example targeted at proteins involved in a disease or in a particular cellular function. Additionally, both LC and HT data can be of different nature: some assays identify proteins that belong to the same complexes, and find mainly stable but potentially indirect interactions (e.g. Affinity purification followed by mass spectrometry [6,7]), while others such as HT-Y2H (highthroughput yeast two-hybrid [8-10]) or PCA (protein complementation assay [1]) search essentially for direct binary interactions that may be transient [11]. Finally, synthetic lethality, genetic suppression and genetic enhancement are examples of genetic interactions, which occur at the phenotypic level and rarely correspond to physical interactions [12]. In this study, we focus on direct binary physical interactions.

Any dataset may contain errors, and particular attention must be paid to false positives (proteins erroneously annotated as interacting). Since interacting proteins in $\mathrm{Y} 2 \mathrm{H}$ are not expressed in their natural cellular context, false positives are restricted here to 'technical' false positives that are due to stochastic or systematic detection method artifacts, and we ignore 'biological' false positives where an interaction is indeed physically possible but not biologically relevant (e.g. if the proteins are never expressed in the same cellular compartment).

\section{Existing estimates}

Since the publication of the first HT-Y2H datasets, several methods for estimating the size of the $S$. cerevisiae interactome have been proposed [5,10,13-18]; it is typically inferred to contain around 20,000 binary interactions, with extreme estimates ranging from 10,000 to 30,500 . These methods are often based on analyses of the HT-Y2H genome-wide screens of the yeast interactome [8-10], and can be broadly divided into two categories. A first class involves the study of the overlap between two or more datasets [14-16,19], usually assumed to follow a hypergeometric distribution. Conceptually these methods differ mainly in their choice of datasets and estimations of error-rates. The second class of methods focuses on a single dataset. Two such methods $[5,13]$ are based on an extrapolation of the number of interactions in an HT [13] or LC [5] subnetwork to the total number of yeast proteins. Another approach applied in the paper reporting the latest HT$\mathrm{Y} 2 \mathrm{H}$ dataset [10] relies on the estimation of their assay's characteristics within a sophisticated framework [3]. This provides detailed information but requires intimate knowledge of the dataset and/or performing additional experiments, hence it may be difficult to accomplish outside the laboratory that produced the data. Finally, Huang and coworkers $[17,18]$ adapted capture-recapture theory and applied it using Interaction Sequence Tag (IST) counts. This is an interesting approach but is only applicable to library-screen-based HT datasets where the number of IST hits is available (a single dataset [8] among those considered in this study). Other estimates based on affinity purification-mass spectrometry data [19] have been proposed but these count indirect interactions and, as this work focuses on the binary interactome, are not directly relevant.

To date, most studies have not explicitly and comprehensively taken into account both LC and HT data. One recent method [10] did use a 'positive reference set' derived from LC data to assess the 'assay sensitivity' of their $\mathrm{Y} 2 \mathrm{H}$ assay, but this dataset represents only a small sample of the available LC interactions and is focused on high confidence rather than wide coverage. However, recent results demonstrate the radically different view that these data offer. For example, the correlation between centrality and lethality, established in 2001 (Jeong et al. [20]) and considered as a given since then, was based on Uetz [9] and LC [21] data; this correlation does not exist [10] in the $\mathrm{Y} 2 \mathrm{H}$-Union dataset (the union of the 3 genome-wide HT-Y2H library screening results [8-10], see Methods, Datasets). One possible explanation lies in the intrinsically different strategies underlying low-throughput and high-throughput data collection (hypothesis-driven versus systematic). Additionally, only $\mathrm{Y} 2 \mathrm{H}$ and PCA have been applied in a high-throughput setting whereas a wide variety of detection methods have been used at low-throughput. Thus high-throughput and low-throughput experiments may have explored different subspaces of the interactome: these two data sources appear complementary, and current estimates of the interactome size are questionable because usually based exclusively on one or the other. Finally, LC data includes highly focused and thorough studies of particular proteins, which may have allowed the identification of some interactions that are intrinsically difficult to detect. This has also never been considered.

We propose here a method for estimating the size of an interactome. It is based on dataset overlap, but takes into account both HT and LC data, as well as interactions that are hard to detect by taking advantage of the 
extensive literature curation efforts undertaken at SGD (the Saccharomyces Genome Database [22]).

\section{Results \\ Method overview}

Our method is based on a comparison between lowthroughput binary physical data curated from the literature (LowBP-LC, obtained from the BioGRID database after filtering), and a binary physical high-throughput dataset (HT-Union, the union of a PCA [1] and three HT-Y2H [8-10] datasets, see Methods). Assuming that HT interactions are randomly drawn within the interactome, and thus independently of their presence in Low $B P-L C$, allows to estimate the interactome size. Indeed, under this assumption, the number of true positive HT interactions included in $L o w B P-L C$ follows a hypergeometric distribution $\mathscr{H}(N, m, n)$, with $N$ the total number of genuine interactions, $m$ the number of true positive LowBP-LC interactions and $n$ the number of true positive HT interactions. Thus, given an estimation of the false-discovery rate $(F D R=F P /(T P+F P)$ with FP and TP the numbers of false positives and true positives, respectively) of each dataset, one can compute the number of genuine interactions in the whole interactome. This is the basis for most methods relying on the overlap between datasets [14-16,19].

However, all assays have their biases and limitations: some interactions may be easy to detect with one assay and difficult or impossible with another. In addition, most HT datasets were obtained with $\mathrm{Y} 2 \mathrm{H}$, but this assay is also widely used in low-throughput studies - it provides support for $53 \%$ of LowBP-LC interactions according to BioGrid evidence codes. It follows that $L o w B P-L C$ is expected to be enriched in interactions that are readily detectable with $\mathrm{Y} 2 \mathrm{H}$. This hypothesis is supported by studying Ito and co-workers' data [8]. Indeed, we used the number of IST hits (interaction sequence tags) for each interaction as an indicator of the difficulty to detect it: interactions with more ISTs are easier to detect, at least in Ito and coworkers' version of the $\mathrm{Y} 2 \mathrm{H}$ protocol. We observed that the number of IST hits is clearly correlated with over-representation in LowBP-LC (See Figure 1 and Methods). As this phenomenon exists with both LowBP-LC and LowBP$L C$-pre 2000 (interactions reported before 2000), it is not due to the fact that low-throughput experiments could have been designed to confirm Ito-Core interactions (HT-Y2H interactions seen at least 3 times in Ito et al. [8], 2001). In addition, although the lower representation observed for interactions with 1 and 2 IST hits is likely partly due to higher FDRs among these interactions, reported as lower confidence in the original article [8], the coverage by $L o w B P-L C$ keeps increasing with the number of ISTs for interactions with 3 or more ISTs. These putative interactions - including any false positives among

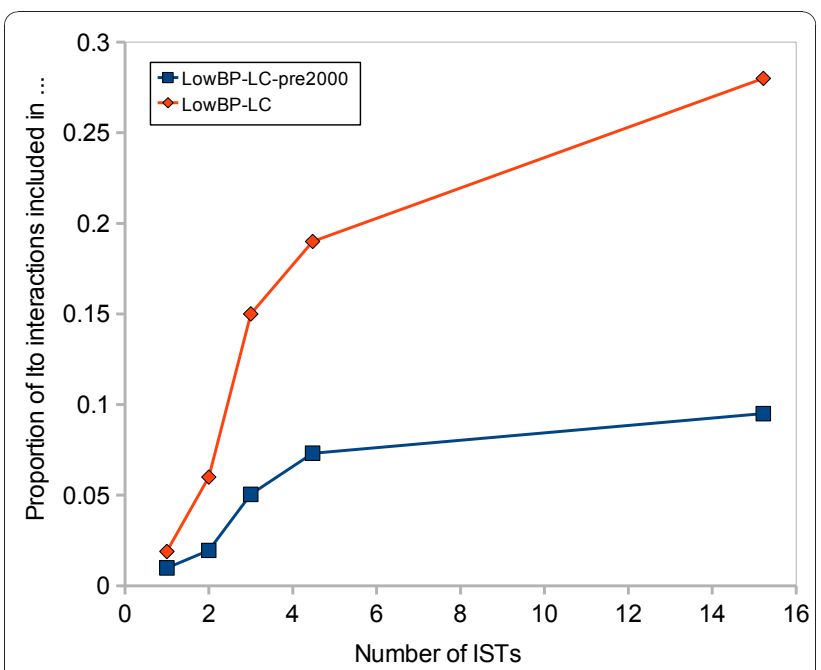

Figure 1 Increased coverage by literature-curated datasets of interactions that are easier to detect by $\mathrm{Y} 2 \mathrm{H}$. The proportion of Ito interactions present in LOWBP-LC and in LOWBP-LC-pre2000 (literature-curated interactions reported before 2000) is plotted as a function of the number of IST hits. Each point represents at least 200 interactions, and the number of IST hits is the weighted mean for these interactions.

them - are well reproducible in this particular experimental system, hence the FDR is not expected to decrease when the number of ISTs increases. We conclude that the presence of an interaction in $L o w B P-L C$ is positively correlated with the ease of finding it by $\mathrm{Y} 2 \mathrm{H}: L o w B P-L C$ is indeed enriched in $\mathrm{Y} 2 \mathrm{H}$-strong interactions. Thus the assumption that HT and LC data are independent subsets of the complete interactome does not hold, and the simple dataset overlap method described above leads to underestimating the interactome size.

Our method can be summarized as follows. In order to alleviate this problem, we restrict the LowBP-LC dataset to interactions involving proteins that have been thoroughly studied: we show that these proteins have likely been subjected to a wider variety of assays, leading to a less biased view of the interactome. We then estimate the FDRs of LowBP-LC and of each HT dataset, using dataset overlap to relate the HT FDRs to one another. Finally, we model the number of HT true positives included in $L o w B P-L C$ restricted to well-studied proteins by a hypergeometric distribution $\mathscr{H}\left(N, m^{\prime}, n\right)$, with $N$ and $n$ as described above and $m$ ' the number of true positive $L o w B P-L C$ interactions involving well-studied proteins (equation (5)). This leads to an estimation of the interactome size $N$.

\section{Taking into account how thoroughly proteins have been studied}

We examined the relation between a protein's degree (i.e. the number of interactions it is involved in) and 
how thoroughly it has been studied, modeled as the number of papers in which the protein has been cited (according to the Saccharomyces Genome Database [23], see Methods). This revealed a strong correlation between these two quantities for the LowBP-LC dataset (Figure 2a): as expected, literature curation has reported many more interactions for highly studied proteins than for poorly studied ones. More surprisingly, a small but significant correlation also exists for the $\mathrm{Y} 2 \mathrm{H}$-Union dataset (Figure $2 \mathrm{~b}$ ). We see no reason why a proteomewide $\mathrm{Y} 2 \mathrm{H}$ screen would identify a larger proportion of the interactions that can be established by well-studied proteins, therefore this observation suggests that the density of the complete interactome is higher for wellstudied proteins than for poorly studied ones. The statistical test is inconclusive with the Tarassov data (Figure 2c). Another unexpected observation is that even for well-studied proteins, LowBP-LC data are far from comprehensive: based on the available $\mathrm{HT}$ data for these proteins, we estimate the false negative rate $(F N R=F N /$ $(T P+F N)$ with TP and FN the numbers of true positives and false negatives) of LowBP-LC restricted to well-studied proteins at approximately $60 \%$ (see Methods and Tables 1 and 2).

\section{Well-studied data comprise interactions that are difficult to detect}

A closer look at the interaction data concerning wellstudied proteins leads to another surprising discovery: HT data covers LowBP-LC much better than it does $L o w B P-L C$ restricted to interactions involving well-studied proteins (Figure 3). Note that this is not due to the fact that $L o w B P-L C$ has a better coverage of the complete interactome restricted to well-studied proteins: indeed, the completeness of LowBP-LC should not affect the proportion of its interactions that are present in an independent subset of the interactome. Thus, we see only two possible explanations.

First, this could be simply because the rate of false positives in $L o w B P-L C$ increases when restricting this dataset to well-studied proteins. Cusick et al. [24] recurated 100 literature-curated yeast interactions, which allows us to invalidate this hypothesis: for these interactions, we found that false positives are not over-represented among LowBP-LC interactions involving wellstudied proteins (well-studied interactions represent $21.4 \%$ of the false positives and $22 \%$ of the true positives, see Methods).

As an alternative explanation, we propose that indepth studies discover interactions that are difficult to detect by most widespread methods, hence are underrepresented in HT datasets. To test this hypothesis, we examined whether the experimental methods used to demonstrate LowBP-LC well-studied interactions
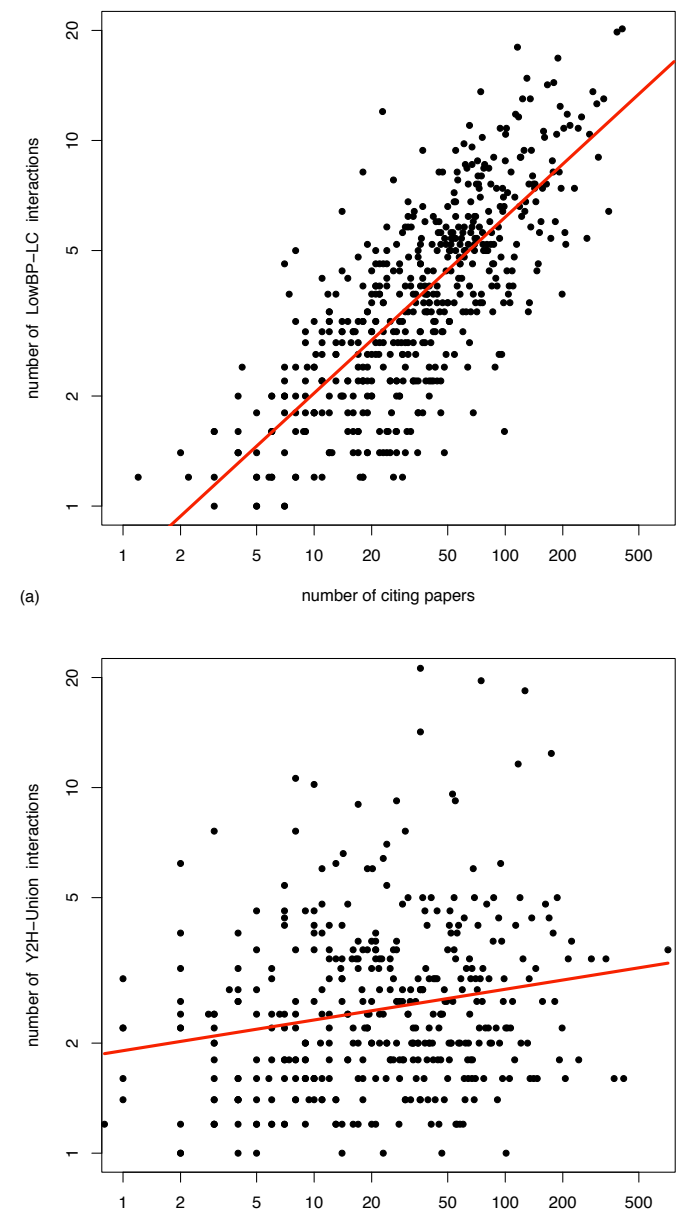

(b) number of citing papers

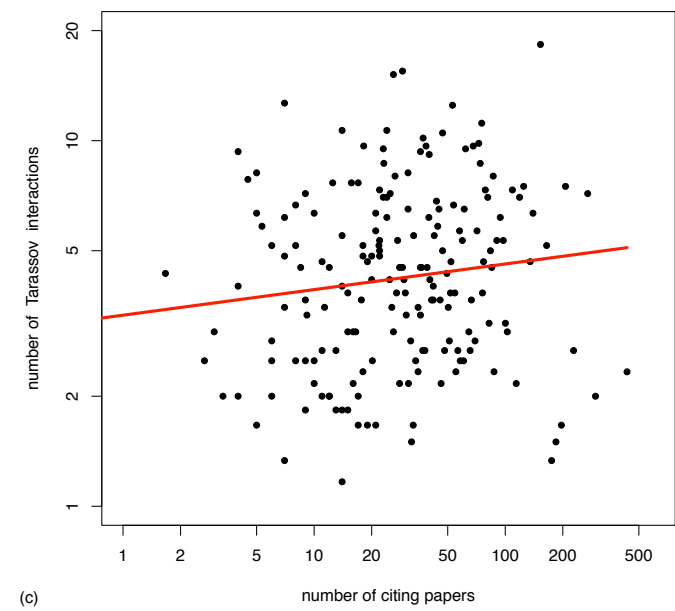

Figure 2 Relation between the level of study and the degree of proteins in various datasets. Log-log scale linear regression between the number of interactions (in the indicated dataset) involving a protein and the number of papers referencing that protein, using binned data (each point represents 5 proteins). (a) LowBP-LC interactions, $R^{2}=0.59, \mathrm{P}=2 \cdot 10^{-103}$, slope $=0.48$. (b) $Y 2 \mathrm{H}$ Union interactions, $R^{2}=0.04, P=1.0 \cdot 10^{-4}$, slope $=0.08$. (c) Tarassov interactions, $R^{2}=0.01, P=0.07$, slope $=0.07$. 
Table 1 Estimated false negative rate of LowBP-LC restricted to interactions involving well-studied proteins.

\begin{tabular}{ccccccc}
\hline Well-studied cutoff & Uetz-Screen & Ito-Core & CCSB-YI1 & Y2H-Union & Tarassov & HT-Union \\
\hline 100 & 0.64 & 0.66 & 0.66 & 0.68 & 0.63 & 0.67 \\
105 & 0.63 & 0.65 & 0.65 & 0.67 & 0.61 & 0.66 \\
110 & 0.64 & 0.66 & 0.65 & 0.67 & 0.61 & 0.66 \\
115 & 0.65 & 0.66 & 0.64 & 0.67 & 0.61 & 0.62 \\
120 & 0.66 & 0.65 & 0.63 & 0.66 & 0.61 & 0.65 \\
125 & 0.66 & 0.66 & 0.65 & 0.67 & 0.62 & 0.66 \\
130 & 0.63 & 0.49 & 0.63 & 0.63 & 0.62 & 0.63 \\
135 & 0.60 & 0.49 & 0.62 & 0.61 & 0.64 & 0.62 \\
140 & 0.60 & 0.47 & 0.62 & 0.61 & 0.64 & 0.61 \\
145 & 0.62 & 0.45 & 0.61 & 0.60 & 0.61 & 0.62 \\
150 & 0.63 & 0.45 & 0.61 & 0.61 &
\end{tabular}

The false negative rate is computed separately with each high-throughput dataset, using a cutoff to consider proteins well-studied ranging from 100 to 150 and a reference FDR for CCSB-YI1 set at 0.25 .

differed significantly from those used to demonstrate other LowBP-LC interactions, using the BioGrid experimental evidence codes. We observed that interactions in the well-studied subset are less frequently supported by $\mathrm{Y} 2 \mathrm{H}$ (down $13.9 \%$ from $58.6 \%$ to $44.7 \%$, p-value $<2.2 \mathrm{e}-$ $16)$, while they are significantly more frequently supported by biochemical activity assays such as those detecting phosphorylation or ubiquitination (Biochemical Activity, up $12.4 \%$ from $11.1 \%$ to $23.5 \%$, p-value < $2.2 \mathrm{e}-16$ ), as well as in vitro assays using purified proteins (Reconstituted Complex, up $8.5 \%$ from $33.5 \%$ to $42 \%$, $\mathrm{p}$-value $=5.5 \mathrm{e}-12)$. Thus well-studied proteins have more often been subjected to labor-intensive interaction detection methods, which may allow the detection of a wider variety of interactions. To sum up, this supports the hypothesis that literature-curated interaction data involving well-studied proteins comprise interactions that, although genuine, are difficult or impossible to detect using labor-efficient methods such as $\mathrm{Y} 2 \mathrm{H}$.

Taking into account the level of study of proteins may thus allow to account for these interactions, hence lead to more accurate estimates of the size of an interactome.

\section{LowBP-LC false positives}

Literature-curated data has been commonly assumed of excellent quality, but a recent study showed that curation errors may not be so infrequent. Cusick et al. [24]

Table 2 Influence of the CCSB-YI1 FDR on the LowBP-LC well-studied false negative rate.

\begin{tabular}{ccccccc}
\hline $\begin{array}{c}\text { CCSB-YI1 } \\
\text { FDR }\end{array}$ & $\begin{array}{c}\text { Uetz- } \\
\text { Screen }\end{array}$ & $\begin{array}{c}\text { Ito- } \\
\text { Core }\end{array}$ & $\begin{array}{c}\text { CCSB- } \\
\text { YI1 }\end{array}$ & $\begin{array}{c}\text { Y2H- } \\
\text { Union }\end{array}$ & Tarassov & $\begin{array}{c}\text { HT- } \\
\text { Union }\end{array}$ \\
\hline 0.15 & 0.67 & 0.66 & 0.65 & 0.68 & 0.57 & 0.66 \\
0.25 & 0.63 & 0.62 & 0.61 & 0.64 & 0.53 & 0.62 \\
0.35 & 0.59 & 0.57 & 0.57 & 0.58 & 0.48 & 0.56
\end{tabular}

The false negative rate of $L O W B P-L C$ restricted to interactions involving wellstudied proteins is computed with the different datasets, when the CCSB-YI1 FDR ranges from 0.15 to 0.35 , using a well-studied cutoff set at 125 . recurated 100 yeast interactions supported by a single paper, assigning a confidence score to each. They reported that $35 \%$ of these interactions were erroneous and that $40 \%$ could be not verified. For this study, we considered that among LowBP-LC-Unique (interactions from $L o w B P-L C$ supported by a single paper, and not found in the HT dataset), $35 \%$ were false positives. The initial report has been debated $[25,26]$ and this may be an overestimate, which would result in our underestimating the interactome size. Interactions reported in more than one paper, or also detected by an HT experiment, were considered true positives.

\section{HT false positives}

The initial mistrust of HT-Y2H assays was largely based on an analysis [27] benchmarking HT datasets against a

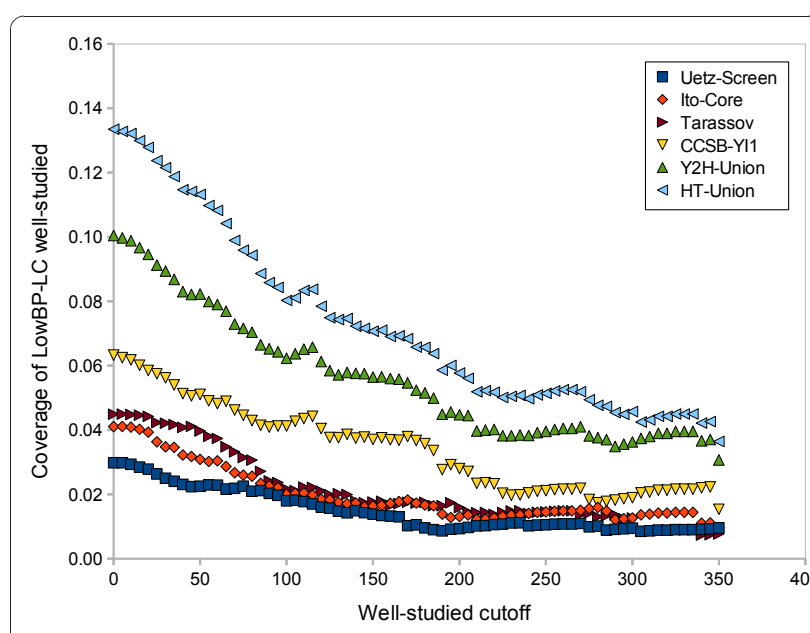

Figure 3 Coverage of LoWBP-LC well-studied by each highthroughput dataset. The proportion of LOWBP-LC interactions involving well-studied proteins that are covered by each $\mathrm{HT}$ dataset is plotted as a function of the 'well-studied cutoff', i.e. the minimum number of papers referencing a protein for it to be considered wellstudied. 
set of protein complexes expanded with the matrix model, and does not seem relevant anymore $[10,18]$. Indeed, after the publication of the first HT-Y2H datasets, several methods estimated their FDRs at 50\% (e.g. $[14,16])$. However, by retesting their own data with orthogonal assays, Yu et al. [10] have estimated the FDR of $C C S B-Y I 1$, their proteome-wide HT-Y2H dataset, at 0-6\%, and showed that Uetz-Screen (the Uetz et al. HT$\mathrm{Y} 2 \mathrm{H}$ library screening result [9]) and Ito-Core are also of high quality. Based on the capture/recapture method, Huang et al. [18] have evaluated the FDR of Ito-Full to $26 \%$. Ito-Full is comprised of all interactions from Ito et al. [8] including those reported as low confidence in the original publication, and is known to have the lowest quality (e.g. $[10,14,28])$. As there is no consensus on the order of magnitude of these FDRs, we decided to apply our method with different FDR values. The CCSB-YI1 FDR is taken ranging from $15 \%$ to $35 \%$ and the other HT FDRs are computed as described below.

We developed a simple method for comparing the FDRs of high-throughput datasets, based on the hypothesis that the Low BP-LC coverage of HT true positives is the same for each HT dataset (see Methods). Under this assumption, we established a simple relation between the FDRs of HT datasets (Methods, equation (1)). However, if some low-throughput experiments were performed to verify interactions reported in high-throughput datasets, an important bias may favor older datasets, which will 'artificially' have more interactions in common with LowBP-LC. This problem can be addressed by restricting $L o w B P-L C$ to interactions reported before 2000 (the publication date of the oldest HT dataset), yielding another dataset called LowBP-LC-pre2000. In fact, Ito-Core and Uetz-Screen (published in 2001 and 2000) have a higher proportion of interactions in common with LowBP-LC than CCSB-YI1 (published in 2008), whereas with LowBP-LC-pre2000, the proportions are similar (Table 3 ). We therefore used LowBP-LC-pre2000 to estimate the HT FDRs. For example, assuming a CCSB-YI1 FDR of $25 \%$, FDRs of Y2H datasets range from $15 \%$ to $25 \%$ (Table 4 ).

Likewise, historical reasons may favor $\mathrm{Y} 2 \mathrm{H}$ over PCA. Indeed, Y2H was proposed in 1989 [29], and has been widely used in low-throughput experiments, whereas PCA was first described in 2000 [30]. We cannot correct for this bias because restricting LowBP-LC to interactions reported before 1989 yields a very small dataset. As a consequence the FDR of $73 \%$ that can be computed for Tarassov (PPIs detected by high-throughput
Table 4 Estimated false discovery rate of each highthroughput dataset.

\begin{tabular}{cccccc}
\hline & CCSB-YI1 & Uetz-Screen & Ito-Core & Tarassov & Ito-Full \\
\hline FDR & 0.25 & 0.15 & 0.21 & 0.73 & 0.76 \\
\hline
\end{tabular}

The FDRs are computed with eq (3), setting the CCSB-YI1 FDR at 0.25. As discussed, the FDR that can be computed for the Tarassov dataset is a rough upper bound.

protein complementation assay [1]) may be largely overestimated and is only a rough upper bound.

\section{Estimating the interactome size}

Starting with the number of LowBP-LC interactions involving well-studied proteins (2572 interactions), we removed the expected number of false positives $(35 \%$ of LowBP-LC-Unique). We then calculated on the one hand the number of interactions, all considered as genuine, in the intersection between the LowBP-LC wellstudied subset and the HT dataset (144 interactions for HT-Union, see Table 5 for the other datasets), and on the other hand the estimated number of true positives in the whole HT dataset, taking into account HT false positives by using the HT FDRs estimated as described above and assuming an FDR of 25\% for CCSB-YI1 ( 2814 true positives in HT-Union, see Table 5 for the other datasets). Taken together, this allows to estimate the size of the binary yeast interactome at $\sim 37,600$ interactions (95\% confidence interval: 32252-43472, constructed with the normal approximation method [31]). Details on the calculation are provided in Methods.

The LowBP-LC well-studied subset was defined with a cutoff (number of referencing papers for a protein to be considered well-studied) of 125 papers, which seems a good compromise between the number of proteins in the subset and how thoroughly they have been studied (Figure 4). The choice of this cutoff or even changes in the HT datasets have little influence on the estimate: it varies between 30,500 and 43,000 interactions, with a cutoff ranging from 100 to 150 and using all the different HT datasets, either singly or merged (Figure 5). Because of the LowBP-LC / HT correlation, which is likely still present even when using the well-studied subset of LowBP-LC, the results presented here may be underestimated. Obviously, increasing the estimated HT FDRs decreases the interactome size (Figure 6), and more precise results could be obtained with better estimates of these FDRs.

Table 3 Proportion of HT interactions included in LowBP-LC-pre2000 and LowBP-LC for the different datasets.

\begin{tabular}{lcccccc}
\hline & Uetz-Screen & Ito-Core & CCSB-YI1 & Y2H-Union & Tarassov & Ito-Full \\
\hline LowBP-LC-pre2000 & 0.0831 & 0.0767 & 0.0734 & 0.0634 & 0.0264 & 0.0235 \\
LowBP-LC & 0.2017 & 0.2254 & 0.1617 & 0.1601 & 0.0746 & 0.0637 \\
\hline
\end{tabular}


Table 5 Calculation steps leading to the interactome size. The well-studied cutoff is set at 125 papers and the CCSB-YI1 FDR at $\mathbf{0 . 2 5}$.

\begin{tabular}{lcccccc}
\hline & Uetz-Screen & Ito-Core & CCSB-YI1 & Y2H-Union & Tarassov & HT-Union \\
\hline LowBP-LC well-studied size & & & & 2572 & & 1909.45 \\
LowBP-LC well-studied TPS & 1905.95 & 1908.4 & 1911.55 & 1916.45 & 746.2 & 2814 \\
HT TPS & 572.4 & 654.2 & 1349.3 & 2171.8 & 38 & 144 \\
HTnLowBP-LC well-studied & 30 & 35 & 72 & 112 & 375 \\
Estimated size & 36366 & 35670 & 35822 & 37163 & 37574 \\
\hline
\end{tabular}

By and large, our estimates are higher than previous ones, which is reasonable as we used all available datasets and took advantage of their complementarity, and we accounted for interactions that are difficult to detect.

\section{Discussion}

As mentioned in the introduction, several methods based on dataset overlap have been proposed for estimating the yeast interactome size [14-16]. The main differences between these methods lie in the error-rate estimations and in the datasets used. While Grigoriev and co-workers [15] consider that false positives and false negatives compensate each other, d'Haeleseer and Church [16] estimate false-discovery rates thanks to the overlap of two HT datasets with a reference LC dataset, and Sprinzak and coworkers' FDR estimation [14] is based on co-localization data. In our method, a reference FDR for one dataset was chosen following a review of the literature, and the overlap between high-throughput and literature-curated data is used to derive the FDRs of other HT datasets from the reference FDR, somewhat similarly to d'Haeleseer and Church. Another important factor for this class of

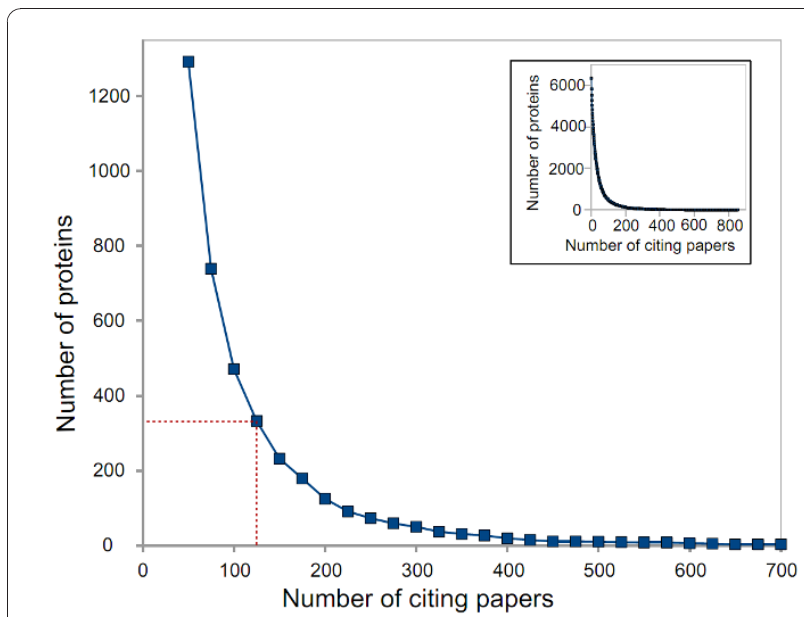

Figure 4 Number of well-studied proteins. The number of proteins in the well-studied subset is plotted as a function of the well-studied cutoff value. The main figure is restricted to proteins cited in at least 50 papers, while the inset shows the complete graph (starting at one paper). The well-studied cutoff value is the minimum number of papers referencing a protein, for this protein to be considered well-studied. methods lies in the choice of datasets, beyond the necessity of selecting appropriate data (e.g. genetic interactions or co-complex membership may not be directly relevant when studying binary physical interactions). While considering only HT datasets [15] restricts the estimation to interactions that can be detected with the HT method, using a gold standard reference set that is assumed errorfree $[14,16]$ is also problematic. In our method carefully selected LC and HT data are combined, taking into account error-rate estimations for each dataset.

The main advantages of our method are the following. First and foremost, by leveraging the available knowledge of how extensively proteins have been studied, our method accounts for interactions that are genuine yet difficult to detect with commonly-used experimental assays. This significantly increases the predicted interactome size, and has never been taken into account. Secondly, it is applicable to any dataset or union of datasets, and it allows to use most of the available data independently of the experimental detection methods.

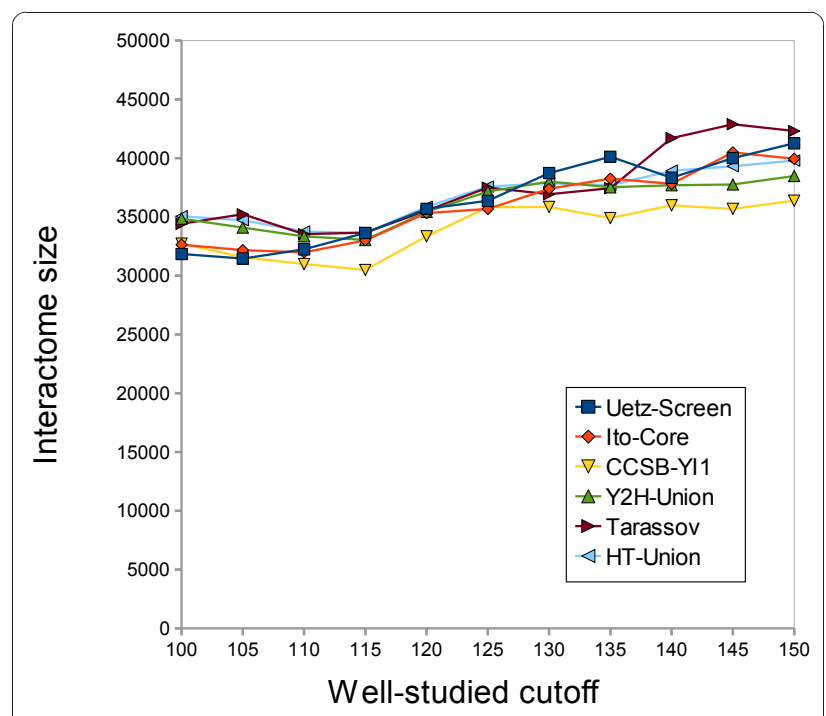

Figure 5 Estimated size of the yeast interactome. The predicted number of binary physical protein-protein interactions that can occur in S. cerevisiae is plotted as a function of the well-studied cutoff value, using each high-throughput dataset and a CCSB-YI1 FDR of 0.25 . The well-studied cutoff value is the minimum number of papers referencing a protein, for this protein to be considered well-studied. 


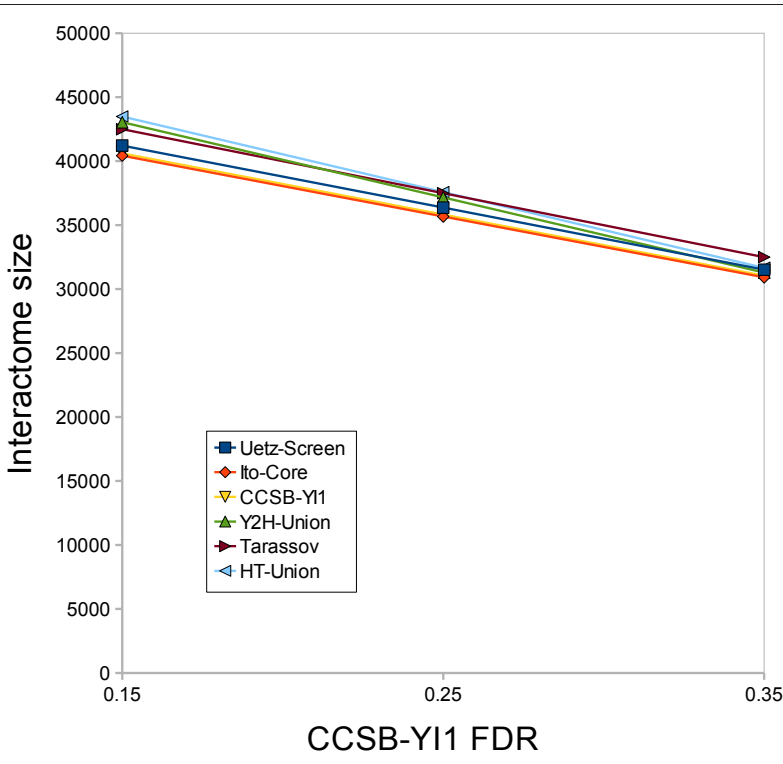

Figure 6 Influence of the CCSB-YI1 FDR on the estimated interactome size. The predicted size of the $S$. cerevisiae interactome is plotted using each high-throughput dataset, when the CCSB-YI7 FDR ranges from 0.15 to 0.35 . The well-studied cutoff (number of papers for a protein to be considered well-studied) is set at 125 papers

Thus, the estimates are easy to update when new datasets become available. Furthermore, our model does not directly rely on a gold standard (i.e. a subset assumed to contain only true positives), which can be difficult to construct and can introduce biases of its own. Likewise, as no dataset is error-free, it is important to consider error rates of both HT and LC datasets.

We have also shown that well-studied proteins appear capable of establishing more interactions than poorly studied ones (Figure $2 \mathrm{~b}$ ). This probably stems from the fact that well connected proteins are more likely to play important roles in diverse cellular functions, and therefore attract more attention from the community. Our method inherently takes into account this bias. In addition, our method is robust with respect to the choice of HT datasets. Contrary to other estimates [13,14], which increase by $90 \%$ and $66 \%$ when substituting datasets (respectively Ito-Full for Uetz and Uetz for Ito-Core), ours only changes by at most $15 \%$ when using different $\mathrm{Y} 2 \mathrm{H}$ datasets (at any given well-studied cutoff). Even when comparing estimates based on data obtained by very different assays ( $\mathrm{Y} 2 \mathrm{H}$ and $\mathrm{PCA})$, the variation remains low (20\%). Lastly, the results presented here are for $S$. cerevisiae, but our method could be applied to other organisms, as long as a genome-wide screen as well as significant literature curation have been performed. A potential weakness of our method is that it relies on overlap between datasets that can be small, which may affect the robustness of the estimates.

\section{Conclusion}

In this work, we have analyzed HT and LC data while considering how thoroughly each protein has been studied. This has provided novel insight into existing interactome datasets: on the one hand, well-studied proteins seem capable of establishing more interactions than poorly studied ones, and on the other hand, in-depth studies of these well-studied proteins have allowed to identify interactions that are difficult to detect. Together with the combined use of LC and HT data, these observations allow to accurately estimate the interactome size. Our results show that the size of interactomes tend to be underestimated, as previous estimates are usually based on only one source of data and do not take into account interactions difficult to detect. No highthroughput technique can detect all interactions, and false negatives are unavoidable [32]. As a consequence, a variety of methods must be considered when working with interactome mapping, and new strategies such as prioritization and smart-pooling should be employed $[4,33,34]$. Extensive efforts will be required before an interactome map can be called 'complete', and until then biological conclusions based on the analysis of available data must be drawn with care.

\section{Methods}

\section{Datasets}

LowBP-LC contains 6, 272 low-throughput binary physical interactions gathered from BIOGRID-ORGANISMSaccharomyces_cerevisiae-3.0.64.tab (downloaded from the BioGRID website) [35]. All papers referencing more than 100 interactions were considered as high-throughput, and their interactions were excluded. Among the remaining interactions, only binary physical data was kept, i.e. interactions whose detection method was by Reconstituted Complex, Two-hybrid, Far Western, Biochemical Activity, Co-crystal Structure, Protein-peptide, PCA or FRET (fluorescence resonance energy transfer).

Ito-Core [8], Uetz-Screen [9], CCSB-YI1 and $Y 2 H$ Union [10] are HT-Y2H datasets: Ito-Core contains the interactions seen at least 3 times by Ito et al., UetzScreen is the Uetz et al. genome-wide library screening result, and $\mathrm{Y} 2 \mathrm{H}$-Union is the union of these two datasets with CCSB-YI1 [10]. All these $\mathrm{Y} 2 \mathrm{H}$ datasets were downloaded from the Center for Cancer Systems Biology website [36]. Ito-Full contains all interactions from Ito et al. [8]. It was downloaded from the Ito Laboratory website [37]. Tarassov are the PPIs detected by highthroughput protein complementation assay [1] (provided as supplementary material in the original publication). HT-Union contains all interactions from all HT datasets.

The level of study of a protein is modeled by the number of papers in which it has been cited, computed from a table of associations between literature and genes 
(downloaded from the Saccharomyces Genome Database [23] on 2010/05/03). Comparing HT FDRs requires to restrict the $L$ ow $B P-L C$ dataset to interactions reported before 2000 in LowBP-LC-pre2000. LowBP-LC-Unique are interactions from $L o w B P-L C$ supported by a single paper, and not found in the considered HT dataset.

Additional file 1 presents the number of interactions and unique proteins in each dataset and intersection of datasets. All datasets are provided in Additional file 2.

\section{The false positive rate of LowBP-LC does not depend on the level of study}

Cusick et al. recurated 100 literature-curated yeast interactions, assigning confidence score for each one: 0 for no confidence, 1 for low confidence or unsubstantiated and 2 for substantiated or high confidence. We therefore considered interactions with a score of 0 to be false positives, and those with a score of 2 to be true positives. We then computed the proportion of these interactions that involve well-studied proteins for each category. Among the 35 false positive interactions and the 25 true positives, respectively $21.4 \%$ and $22 \%$ involve a well-studied protein.

\section{LowBP-LC false negatives}

Hypothesizing that HT well-studied and LowBP-LC well-studied are independent allows to estimate the expected number of genuine interactions involving wellstudied proteins, and thus the LowBP-LC well-studied false negative rate:

$$
F N R_{L o w B P-L C_{W S}}=1-\frac{T P_{H T_{W S} \cap L o w B P-L C}}{T P_{H T_{W S}}}
$$

with $T P_{H T_{W S}}$ the estimated number of true positives in $H T_{W S}$, the HT dataset restricted to interactions involving well-studied proteins, and $T P_{H T_{W S} \cap L o w B P-L C}$ the number of true positives within the intersection between $H T_{W S}$ and LowBP-LC.

\section{A relation between $\mathrm{HT}$ FDRs}

To decrease the potential correlation between LowBP$L C$ and older HT-Y2H datasets due to recent studies that could have been designed to confirm HT interactions, the LowBP-LC dataset used for the FDR calculations contains only interactions reported in publications published before 2000 (publication date of the oldest HT dataset). Consider two HT datasets, denoted 1 and 2 (e.g. Ito-Core and CCSB-YI1), each partitioned into three subsets $A, B$ and $C$, respectively the true positives included in LowBP-LC-pre2000, the true positives not included in LowBP-LC-pre2000 and the false positives. We consider that HT interactions also present in LowBP-LC-pre2000 are true positives (because detected by two independent methods). Therefore, LowBP-LCpre 2000 and $C$ are disjoint. Hypothesizing that the proportion of true positive HT interactions in LowBP-LCpre2000 is independent of the HT dataset yields:

$$
\frac{A_{1}}{B_{1}}=\frac{A_{2}}{B_{2}} .
$$

The proportion of HT interactions included in LowBP-LC-pre2000 $(A /(A+B+C))$ can be easily computed, and denoting $\alpha$ as

$$
\frac{A_{1}}{A_{1}+B_{1}+C_{1}}=\alpha \cdot \frac{A_{2}}{A_{2}+B_{2}+C_{2}},
$$

we obtain a relation between the false-discovery rates of the two datasets, defined as $F D R=\frac{C}{A+B+C}$

$$
F D R_{1}=\alpha \cdot F D R_{2}+1-\alpha .
$$

In the rest of this work, we always use CCSB-YI1 for set 2 .

\section{Computing the interactome size \\ Parameters}

- $H T$ : the $H T$ dataset used.

- Well-studied cutoff: number of papers referencing a protein to consider it well-studied.

- $F D R_{Y I 1}$ : the CCSB-YI1 FDR, required to compute the FDRs of other HT datasets.

\section{Abbreviations and notations}

- WS: well-studied.

- $T P_{\text {Datase }}:$ estimated number of true positives in Dataset.

- |Dataset|: size of Dataset.

- Is: Interactome size.

\section{HT true positives}

- The FDR of Ito-Core, Uetz-Screen and Tarassov is calculated from the FDR of CCSB-YI1 as described in Methods, A relation between HT FDRs:

$$
F D R_{H T}=\alpha \cdot F D R_{Y I I}+1-\alpha
$$

- The number of HT true positives is then computed as follows:

$$
T P_{H T}=|H T|-\sum\left|H T_{i}\right| * F D R_{H T_{i}}
$$

where $H T_{i}$ iterates over the datasets making up HT for union datasets (e.g. for Y2H-Union: Ito-Core, Uetz-Screen and CCSB-YI1), or HT itself for individual datasets such as Ito-Core. 
LowBP-LC true positives

$$
\begin{aligned}
& T P_{\text {LowBP-LC }_{\mathrm{WS}}}= \\
& \quad \mid \text { LowBP-LC }_{\mathrm{WS}}|-35 \% \cdot| \text { LowBP-LC-Unique }_{\mathrm{WS}} \mid
\end{aligned}
$$

Where LowBP-LC-Unique WS $_{\text {S }}$ contains LowBP-LC interactions involving well-studied proteins, supported by a single paper and not in the HT dataset.

\section{True positives in the intersection}

All interactions in the intersection between HT and LowBP-LC are considered true positive, so:

$$
T P_{H T \cap L o w B P-L C_{W S}}=\left|H T \cap L o w B P-L C_{W S}\right| .
$$

\section{Interactome size}

The hypergeometric assumption discussed in Results, Method overview leads to:

$$
I s=\frac{T P_{H T} \cdot T P_{L o w B P-L C}}{T P_{H T}}
$$

with $T P_{H T}, T P_{L o w B P-L C}$ and $T P_{H T \cap L o w B P-L C_{W S}}$ computed as described above (equations (2), (4) and (4)).

This can be expanded to:

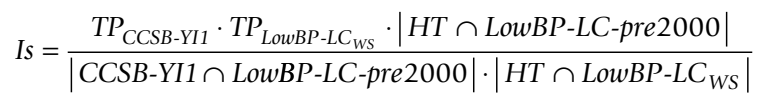

This expanded form allows to study the influence of the various parameters. All relevant scripts are distributed under the GNU General Public License in Additional file 2 .

\section{Presence of ' $\mathrm{Y} 2 \mathrm{H}$-strong' interactions in LowBP-LC}

To examine whether interactions that are more easily detected in $\mathrm{Y} 2 \mathrm{H}$ are also overrepresented in LowBP-LC, we gathered Ito-Full hits and binned them by increasing number of ISTs, each bin containing at least 200 interactions. Each bin is represented by the weighted mean of the number of ISTs, and the proportion of interactions present in LowBP-LC. In order not to separate interactions with the same number of ISTs, some bins (particularly single hits) are larger than others. This analysis is performed both with the complete LowBP-LC and with LowBP-LC-pre2000 (LowBP-LC interactions reported before 2000)(Figure 1).

\section{Additional material}

Additional file 1: Number of interactions and proteins in each dataset. Additional file 1 presents the number of interactions and unique proteins in each dataset and intersection of datasets.
Additional file 2: Datasets and scripts. Additional file 2 is an archive that includes all scripts, distributed under an open source license, as well as all datasets used in this study.

\section{Abbreviations}

PPI: protein-protein interaction; LC: literature-curated; HT: high-throughput; $\mathrm{Y} 2 \mathrm{H}$ : yeast two-hybrid; PCA: protein complementation assay; FDR: falsediscovery rate; IST: interaction sequence tag.

\section{Acknowledgements}

This work was supported by a grant from the Region Rhone-Alpes (to NTM).

\section{Authors' contributions}

NTM designed the study. LS implemented the method and performed the analyses. Both authors drafted and revised the manuscript. They have read and approved its final version.

Received: 11 June 2010 Accepted: 21 December 2010 Published: 21 December 2010

\section{References}

1. Tarassov K, Messier V, Landry CR, Radinovic S, Molina MMS, Shames I, Malitskaya $Y$, Vogel J, Bussey H, Michnick SW: An in vivo map of the yeast protein interactome. Science 2008, 320:1465-1470.

2. Han JDJ, Dupuy D, Bertin N, Cusick ME, Vidal M: Effect of sampling on topology predictions of protein-protein interaction networks. Nat Biotechnol 2005, 23:839-844

3. Venkatesan K, Rual JF, Vazquez A, StelzI U, Lemmens I, Hirozane-Kishikawa T, Hao T, Zenkner M, Xin X, Goh Kl, Yildirim MA, Simonis N, Heinzmann K, Gebreab F, Sahalie JM, Cevik S, Simon C, de Smet AS, Dann E, Smolyar A, Vinayagam A, Yu H, Szeto D, Borick H, Dricot A, Klitgord N, Murray RR, Lin C, Lalowski M, Timm J, Rau K, Boone C, Braun P, Cusick ME, Roth FP, Hill DE, Tavernier J, Wanker EE, Barabasi AL, Vidal M: An empirical framework for binary interactome mapping. Nat Methods 2008, 6:83-90.

4. Schwartz AS, Yu J, Gardenour KR, Finley RL Jr, Ideker T: Cost-effective strategies for completing the interactome. Nat Methods 2009, 6:55-61.

5. Reguly T, Breitkreutz A, Boucher L, Breitkreutz BJ, Hon GC, Myers CL, Parsons A, Friesen $H$, Oughtred R, Tong A, Stark C, Ho Y, Botstein D, Andrews B, Boone C, Troyanskya OG, Ideker T, Dolinski K, Batada NN, Tyers $\mathrm{M}$ : Comprehensive curation and analysis of global interaction networks in Saccharomyces cerevisiae. J Biol 2006, 5:11.

6. Gavin AC, Aloy P, Grandi P, Krause R, Boesche M, Marzioch M, Rau C, Jensen $L$, Bastuck S, Dümpelfeld B, Edelmann A, Heurtier MA, Homan V, Hoefert C, Klein K, Hudak M, Michon AM, Schelder M, Schirle M, Remor M, Rudi T, Hooper S, Bauer A, Bouwmeester T, Casari G, Drewes G, Neubauer G, Rick JM, Kuster B, Bork P, Russell RB, Superti-Furga G: Proteome survey reveals modularity of the yeast cell machinery. Nature 2006, 440:631-636.

7. Krogan NJ, Cagney G, Yu H, Zhong G, Guo X, lgnatchenko A, Li J, Pu S, Datta N, Tikuisis AP, Punna T, Peregrín-Alvarez JM, Shales M, Zhang X, Davey M, Robinson MD, Paccanaro A, Bray JE, Sheung A, Beattie B, Richards DP, Canadien V, Lalev A, Mena F, Wong P, Starostine A, Canete MM, Vlasblom J, Wu S, Orsi C, Collins SR, Chandran S, Haw R, Rilstone JJ, Gandi K, Thompson NJ, Musso G, St Onge P, Ghanny S, Lam MH, Butland G, Altaf-UI AM, Kanaya S, Shilatifard A, O'Shea E, Weissman JS, Ingles CJ, Hughes TR, Parkinson J, Gerstein M, Wodak SJ, Emili A, Greenblatt JF: Global landscape of protein complexes in the yeast Saccharomyces cerevisiae. Nature 2006, 440:637-643.

8. Ito T, Chiba T, Ozawa R, Yoshida M, Hattori M, Sakaki Y: A comprehensive two-hybrid analysis to explore the yeast protein interactome. Proc Natl Acad Sci USA 2001, 98:4569-4574.

9. Uetz P, Giot L, Cagney G, Mansfield TA, Judson RS, Knight JR, Lockshon D, Narayan V, Srinivasan M, Pochart P, Qureshi-Emili A, Li Y, Godwin B, Conover D, Kalbfleisch T, Vijayadamodar G, Yang M, Johnston M, Fields S, Rothberg JM: A comprehensive analysis of protein-protein interactions in Saccharomyces cerevisiae. Nature 2000, 403:623-627. 
10. Yu H, Braun P, Yildirim MA, Lemmens I, Venkatesan K, Sahalie J, HirozaneKishikawa T, Gebreab F, Li N, Simonis N, Hao T, Rual JF, Dricot A, Vazquez A, Murray RR, Simon C, Tardivo L, Tam S, Svrzikapa N, Fan C, de Smet AS, Motyl A, Hudson ME, Park J, Xin X, Cusick ME, Moore T, Boone C, Snyder M, Roth FP, Barabási AL, Tavernier J, Hill DE, Vidal M: High-quality binary protein interaction map of the yeast interactome network. Science 2008, 322:104-110.

11. Vinayagam A, Stelzl U, Wanker EE: Repeated two-hybrid screening detects transient protein-protein interactions. Theor Chem Acc 2010, 125:613-619.

12. Costanzo M, Baryshnikova A, Bellay J, Kim Y, Spear ED, Sevier CS, Ding H, Koh JLY, Toufighi K, Mostafavi S, Prinz J, St Onge RP, VanderSluis B, Makhnevych T, Vizeacoumar FJ, Alizadeh S, Bahr S, Brost RL, Chen Y, Cokol M, Deshpande R, Li Z, Lin ZY, Liang W, Marback M, Paw J, San Luis BJ, Shuteriqi E, Tong AH, van Dyk N, Wallace IM, Whitney JA, Weirauch MT, Zhong G, Zhu H, Houry WA, Brudno M, Ragibizadeh S, Papp B, Pal C, Roth FP, Giaever G, Nislow C, Troyanskaya OG, Bussey H, Bader GD, Gingras AC, Morris QD, Kim PM, Kaiser CA, Myers CL, Andrews BJ, Boone C: The Genetic Landscape of a Cell. Science 2010, 327:425.

13. Stumpf MPH, Thorne T, de Silva E, Stewart R, An HJ, Lappe M, Wiuf C: Estimating the size of the human interactome. Proc Natl Acad Sci USA 2008, 105:6959-6964.

14. Sprinzak E, Sattath S, Margalit H: How Reliable are Experimental ProteinProtein Interaction Data? J Mol Biol 2003, 327:919-923.

15. Grigoriev $A$ : On the number of protein-protein interactions in the yeast proteome. Nucleic Acids Res 2003, 31:4157-4161.

16. D'haeseleer P, Church GM: Estimating and improving protein interaction error rates. Proc IEEE Comput Syst Bioinform Conf 2004, 216-23.

17. Huang $H$, Jedynak BM, Bader JS: Where have all the interactions gone? Estimating the coverage of two-hybrid protein interaction maps. PLoS Comput Biol 2007, 3:e214.

18. Huang $H$, Bader JS: Precision and recall estimates for two-hybrid screens. Bioinformatics 2009, 25:372-378.

19. Hart GT, Ramani AK, Marcotte EM: How complete are current yeast and human protein-interaction networks? Genome Biol 2006, 7:120.

20. Jeong $H$, Mason SP, Barabasi AL, Oltvai ZN: Lethality and centrality in protein networks. Nature 2001, 411:41-42

21. Xenarios I, Rice DW, Salwinski L, Baron MK, Marcotte EM, Eisenberg D: DIP: the database of interacting proteins. Nucleic Acids Res 2000, 28:289-291.

22. Cherry JM, Adler C, Ball C, Chervitz SA, Dwight SS, Hester ET, Jia Y, Juvik G, Roe TY, Schroeder M, Weng S, Botstein D: SGD: Saccharomyces genome database. Nucleic Acids Res 1998, 26:73.

23. SGD project. "Saccharomyces Genome Database". [http://downloads. yeastgenome.org/literature_curation/gene_literature.tab].

24. Cusick ME, Yu H, Smolyar A, Venkatesan K, Carvunis AR, Simonis N, Rual JF, Borick H, Braun P, Dreze M, Vandenhaute J, Galli M, Yazaki J, Hill DE, Ecker JR, Roth FP, Vidal M: Literature-curated protein interaction datasets. Nat Methods 2009, 6:39-46.

25. Salwinski L, Licata L, Winter A, Thorneycroft D, Khadake J, Ceol A, Aryamontri AC, Oughtred R, Livstone M, Boucher L, Botstein D, Dolinski K, Berardini T, Huala E, Tyers M, Eisenberg D, Cesareni G, Hermjakob H: Recurated protein interaction datasets. Nat Methods 2009, 6:860-861.

26. Cusick ME, Yu H, Smolyar A, Venkatesan K, Carvunis AR, Simonis N, Rual JF, Borick H, Braun P, Dreze M, Vandenhaute J, Galli M, Yazaki J, Hill DE, Ecker JR, Roth FP, Vidal M: Addendum: Literature-curated protein interaction datasets. Nat Methods 2009, 6:934-935.

27. von Mering C, Krause R, Snel B, Cornell M, Oliver SG, Fields S, Bork P: Comparative assessment of large-scale data sets of protein-protein interactions. Nature 2002, 417:399-403.

28. Lee I, Date SV, Adai AT, Marcotte EM: A probabilistic functional network of yeast genes. Science 2004, 306:1555-1558.

29. Fields S, Song O: A novel genetic system to detect protein-protein interactions. Nature 1989, 340:245-246.

30. Michnick SW, Remy I, Campbell-Valois FX, Vallee-Belisle A, Pelletier JN: Detection of protein-protein interactions by protein fragment complementation strategies. Methods Enzymol 2000, 328:208.

31. Sahai $H$, Khurshid $A$ : A note on confidence intervals for the hypergeometric parameter in analyzing biomedical data. Comput Biol Med 1995, 25:35-38.

32. Braun P, Tasan M, Dreze M, Barrios-Rodiles M, Lemmens I, Yu H, Sahalie JM, Murray RR, Roncari L, De Smet AS, Venkatesan K, Rual JF, Vandenhaute J, Cusick ME, Pawson T, Hill DE, Tavernier J, Wrana JL, Roth FP, Vidal M: An experimentally derived confidence score for binary protein-protein interactions. Nat Methods 2009, 6:91-97.

33. Xin X, Rual JF, Hirozane-Kishikawa T, Hill DE, Vidal M, Boone C, ThierryMieg N: Shifted Transversal Design smart-pooling for high coverage interactome mapping. Genome Res 2009, 19:1262.

34. Aryee MJA, Quackenbush J: An Optimized Predictive Strategy for Interactome Mapping. J Proteome Res 2008, 7:4089-4094.

35. Stark C, Breitkreutz BJ, Reguly T, Boucher L, Breitkreutz A, Tyers M: BioGRID: a general repository for interaction datasets. Nucleic Acids Res 2006, 34: D535.

36. Center for Cancer Systems Biology. [http://interactome.dfci.harvard.edu/ S_cerevisiae/index.php?page=download].

37. Ito Laboratory. [http://itolab.cb.k.u-tokyo.ac.jp/Y2H/full_data.txt].

doi:10.1186/1471-2105-11-605

Cite this article as: Sambourg and Thierry-Mieg: New insights into protein-protein interaction data lead to increased estimates of the $S$. cerevisiae interactome size. BMC Bioinformatics 2010 11:605.

\section{Submit your next manuscript to BioMed Central and take full advantage of:}

- Convenient online submission

- Thorough peer review

- No space constraints or color figure charges

- Immediate publication on acceptance

- Inclusion in PubMed, CAS, Scopus and Google Scholar

- Research which is freely available for redistribution

Submit your manuscript at www.biomedcentral.com/submit
C) Biomed Central 